University of Nebraska - Lincoln

DigitalCommons@University of Nebraska - Lincoln

Faculty Publications from Nebraska Center for

Materials and Nanoscience, Nebraska Center Materials and Nanoscience

for (NCMN)

2020

\title{
Chiral Magnetism and High-Temperature Skyrmions in B20-Ordered Co-Si
}

\section{Balamurugan Balasubramanian}

Priyanka Manchanda

Rabindra Pahari

Zhen Chen

Wenyong Zhang

See next page for additional authors

Follow this and additional works at: https://digitalcommons.unl.edu/cmrafacpub

Part of the Atomic, Molecular and Optical Physics Commons, Condensed Matter Physics Commons, Engineering Physics Commons, and the Other Physics Commons

This Article is brought to you for free and open access by the Materials and Nanoscience, Nebraska Center for (NCMN) at DigitalCommons@University of Nebraska - Lincoln. It has been accepted for inclusion in Faculty Publications from Nebraska Center for Materials and Nanoscience by an authorized administrator of DigitalCommons@University of Nebraska - Lincoln. 


\section{Authors}

Balamurugan Balasubramanian, Priyanka Manchanda, Rabindra Pahari, Zhen Chen, Wenyong Zhang, Shah R. Valloppilly, Xingzhong Li, Anandakumar Sarella, Lanping Yue, Ahsan Ullah, Pratibha Dev, David A. Muller, Ralph Skomski, George C. Hadjipanayis, and David J. Sellmyer 


\title{
Chiral Magnetism and High-Temperature Skyrmions in B20-Ordered Co-Si
}

\author{
Balamurugan Balasubramanian $\odot,{ }^{1,2, *}$ Priyanka Manchanda, ${ }^{3}$ Rabindra Pahari $\odot,{ }^{1,2}$ Zhen Chen $\odot,{ }^{4}$ Wenyong Zhang, ${ }^{1,2}$ \\ Shah R. Valloppilly, ${ }^{1}$ Xingzhong Li, ${ }^{1}$ Anandakumar Sarella $\odot,{ }^{1}$ Lanping Yue $\odot,{ }^{1,2}$ Ahsan Ullah $\odot,{ }^{1,2}$ Pratibha Dev, ${ }^{3}$ \\ David A. Muller, ${ }^{4}$ Ralph Skomski, ${ }^{1,2}$ George C. Hadjipanayis, ${ }^{5}$ and David J. Sellmyer ${ }^{1,2, \uparrow}$ \\ ${ }^{1}$ Nebraska Center for Materials and Nanoscience, University of Nebraska, Lincoln, Nebraska 68588, USA \\ ${ }^{2}$ Department of Physics and Astronomy, University of Nebraska, Lincoln, Nebraska 68588, USA \\ ${ }^{3}$ Department of Physics and Astronomy, Howard University, Washington, DC 20059, USA \\ ${ }^{4}$ School of Applied and Engineering Physics, Cornell University, Ithaca, New York 14853, USA \\ ${ }^{5}$ Department of Physics and Astronomy, University of Delaware, Newark, Delaware 19716, USA
}

(Received 17 June 2019; accepted 3 January 2020; published 6 February 2020)

\begin{abstract}
Magnets with chiral crystal structures and helical spin structures have recently attracted much attention as potential spin-electronics materials, but their relatively low magnetic-ordering temperatures are a disadvantage. While cobalt has long been recognized as an element that promotes high-temperature magnetic ordering, most Co-rich alloys are achiral and exhibit collinear rather than helimagnetic order. Crystallographically, the B20-ordered compound $\mathrm{CoSi}$ is an exception due to its chiral structure, but it does not exhibit any kind of magnetic order. Here, we use nonequilibrium processing to produce B20-ordered $\mathrm{Co}_{1+x} \mathrm{Si}_{1-x}$ with a maximum Co solubility of $x=0.043$. Above a critical excess-Co content $\left(x_{c}=0.028\right)$, the alloys are magnetically ordered, and for $x=0.043$, a critical temperature $T_{c}=328 \mathrm{~K}$ is obtained, the highest among all B20-type magnets. The crystal structure of the alloy supports spin spirals caused by Dzyaloshinskii-Moriya interactions, and from magnetic measurements we estimate that the spirals have a periodicity of about $17 \mathrm{~nm}$. Our density-functional calculations explain the combination of high magneticordering temperature and short periodicity in terms of a quantum phase transition where excess-cobalt spins are coupled through the host matrix.
\end{abstract}

DOI: 10.1103/PhysRevLett.124.057201

Alloys crystallizing in the cubic B20 structure (prototype $\mathrm{FeSi}$ ) are among the most fascinating materials in science and technology [1-5]. First, their noncentrosymmetric space group, $P 2_{1} 3$, is achiral due to the neutral $2_{1}$ or $180^{\circ}$ screw axis, which does not distinguish between leftand right-handed screws [6]. However, it allows the incorporation of chiral building blocks (motifs), which makes the B20 structure, Fig. 1(a), chiral [6]. In magnetic B20 crystals, such as $\mathrm{MnSi}$, this chirality leads to nonzero Dzyaloshinskii-Moriya (DM) interactions that create spin spirals and supports skyrmions with broken helicity $[1,2,4,7,8]$. Second, "nonmagnetic" (paramagnetic or diamagnetic) B20 alloys are unique in the sense that they exhibit intriguing band-structure features at or near the Fermi level, leading to "new-fermion" topological quantum chirality [9-11].

A limitation of existing B20 magnets is their low magnetic-ordering temperatures (critical temperatures) $T_{c}$ of less than $300 \mathrm{~K}$, which precludes their use in roomtemperature applications. Examples are $\mathrm{MnSi}(30 \mathrm{~K})$, MnGe (170 K), and FeGe (278 K) [1]. Some B20 alloys are not even ordered at zero temperature, including $\mathrm{CoSi}$ and FeSi $[1,12,13]$. However, cobalt generally exhibits a trend towards ferromagnetism, and $\mathrm{CoSi}$ nanowires having diameters of the order of $50 \mathrm{~nm}$ actually exhibit a small net moment due to spin-polarized Co atoms near the surface $[14,15]$. It is therefore tempting to use a small amount $x$ of excess $\mathrm{Co}$ to replace $\mathrm{Si}$ and to realize a magnetically ordered $\mathrm{Co}\left(\mathrm{Si}_{1-x} \mathrm{Co}_{x}\right)$ or simply $\mathrm{Co}_{1+x} \mathrm{Si}_{1-x}$. Unfortunately, the solid solubility of Co in CoSi is limited to $x \leq 0.02$, yielding a nonmagnetic alloy with the nominal composition $\mathrm{Co}_{1.02} \mathrm{Si}_{0.98}$ [16,17]. In this Letter, we use a nonequilibrium rapid-quenching method to create magnetically ordered polycrystalline $\mathrm{Co}_{1+x} \mathrm{Si}_{1-x}$ with a maximum Co solubility of $x=0.043$ (see Supplemental Material S0 for methods [18]), investigate the onset of magnetism in the system using experiments and density-functional theory, and show a high $T_{c}=328 \mathrm{~K}$ with relatively small periodicity for $\mathrm{Co}_{1.043} \mathrm{Si}_{0.957}$.

We have obtained single-phase B20-ordered $\mathrm{Co}_{1+x} \mathrm{Si}_{1-x}$ for Co contents of up to $x=0.043$. Figure 1(b) shows the experimental x-ray diffraction (XRD) patterns, which are indexed to the cubic B20 structure and compared with Rietveld fittings (black curves). The fittings, which include the replacement of $\mathrm{Si}$ by excess-Co atoms, are in good agreement with the experimental XRD patterns. The lattice constant increases linearly with the Co content, Fig. 1(c), confirming that our alloy is a substitutional alloy of the solid-solution type and obeys Vegard's law in a way consistent with the atomic radii of $\mathrm{Si}$ and $\mathrm{Co}$. 


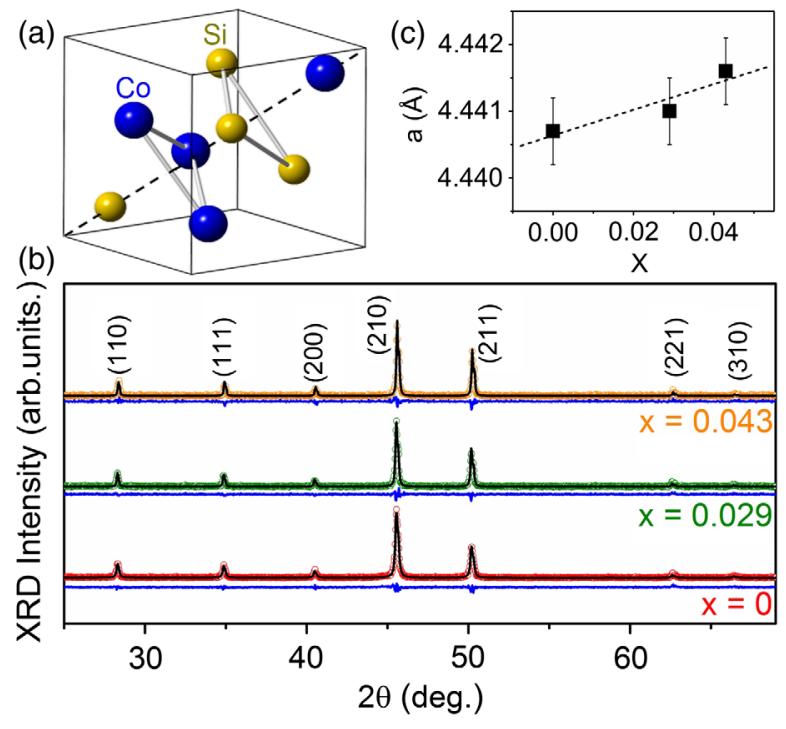

FIG. 1. Crystal structure of B20-ordered $\mathrm{Co}_{1+x} \mathrm{Si}_{1-x}$ : (a) unit cell of equiatomic $\mathrm{CoSi}$ and (b) experimental x-ray diffraction patterns for several values of $x$ (open circles), where the black and blue curves correspond to the simulated XRD patterns using Rietveld analysis based on the B20 structure and difference between the experimental and simulated patterns, respectively. (c) Lattice parameter $(a)$, where the dashed line is the linear fit to the data (squares).

The transmission-electron microscope image in Fig. 2(a) shows the nanostructure for $x=0.043$. The annular brightfield scanning transmission electron microscope (STEM) image is shown in Fig. 2(b), and is composed of Si (gray), Co (black), and $\mathrm{Co}+\mathrm{Si}$ (combination of black and gray) atomic columns in an agreement with the B20 structural model (see Supplemental Material S1 [18]). The Fourier transform (diffractogram) in Fig. 2(c) is also indexed to the B20 structure along the [111] zone axis. A high-angle annular dark-field STEM analysis shows a uniform distribution of $\mathrm{Co}$ and $\mathrm{Si}$ across the sample (Supplemental Material Fig. S2).

All Co and Si sites in the chiral B20 structure [Fig. 1(a)] have the Wyckoff symbols $4 a$ but differ by the parameters $u_{\mathrm{Co}}$ and $u_{\mathrm{Si}} \approx 1-u_{\mathrm{Co}}$. There are two types of structural chirality (helicity), namely left-handed crystals (LHC) when $u_{\mathrm{Co}}<u_{\mathrm{Si}}$ and right-handed crystals (RHC) when $u_{\mathrm{Co}}>u_{\mathrm{Si}}$ [[8,19-21], Supplemental Material S0]. Our XRD analysis indicates that our melt-spun samples contain both left-handed $\left(u_{\mathrm{Co}}=0.148\right.$ and $\left.u_{\mathrm{Si}}=0.84\right)$ and righthanded $\left(u_{\mathrm{Co}}=0.873\right.$ and $\left.u_{\mathrm{Si}}=0.16\right)$ crystallites and that the LHC:RHC ratio is about 53:47.

Figure 3 shows magnetic properties for the three samples ( $x=0,0.029$, and 0.043). The field (a) and temperature (b) dependences of the magnetization show that the samples with $x=0.029$ and 0.043 are magnetically ordered. By contrast, for $x=0$, the $M(H)$ curves in Fig. 3(a) and the $M(T)$ curve in Fig. S3(a) are typical of paramagnetism. The low-temperature magnetizations in a

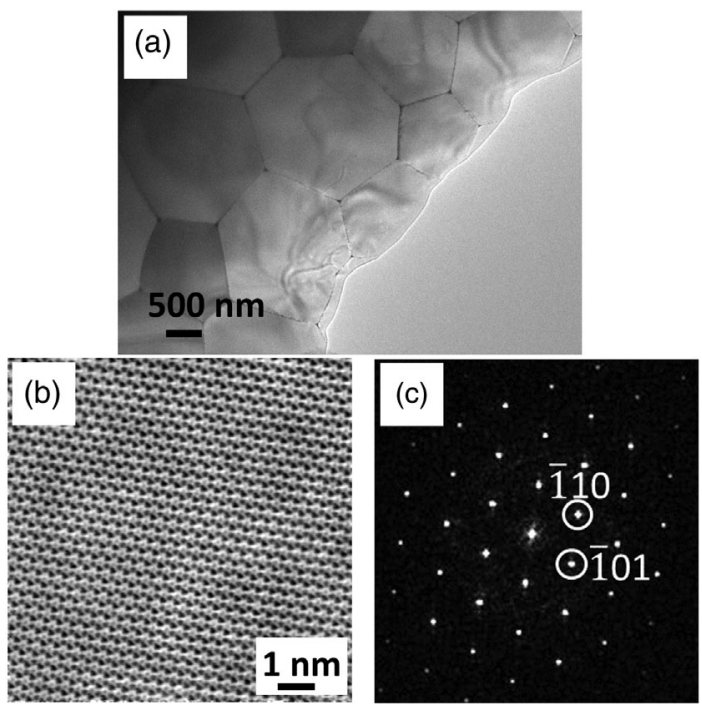

FIG. 2. Structure of the sample with the highest excess-Co content ( $x=0.043)$ : (a) TEM image, (b) annular bright-field scanning transmission-electron microscope image, and (c) the corresponding diffractogram indexed to the cubic B20 structure along the [111] zone axis.

field of $70 \mathrm{kOe}$ are $0.003 \mu_{B} / \mathrm{Co}\left(1.3 \mathrm{emu} / \mathrm{cm}^{3}\right)$ for $x=0.029$ [Fig. S3(b)] and $0.11 \mu_{B} /$ Co $\left(44.6 \mathrm{emu} / \mathrm{cm}^{3}\right)$ for $x=0.043$. More generally $M(H)$ and $M(T)$ curves for helimagnetic materials look similar to those of
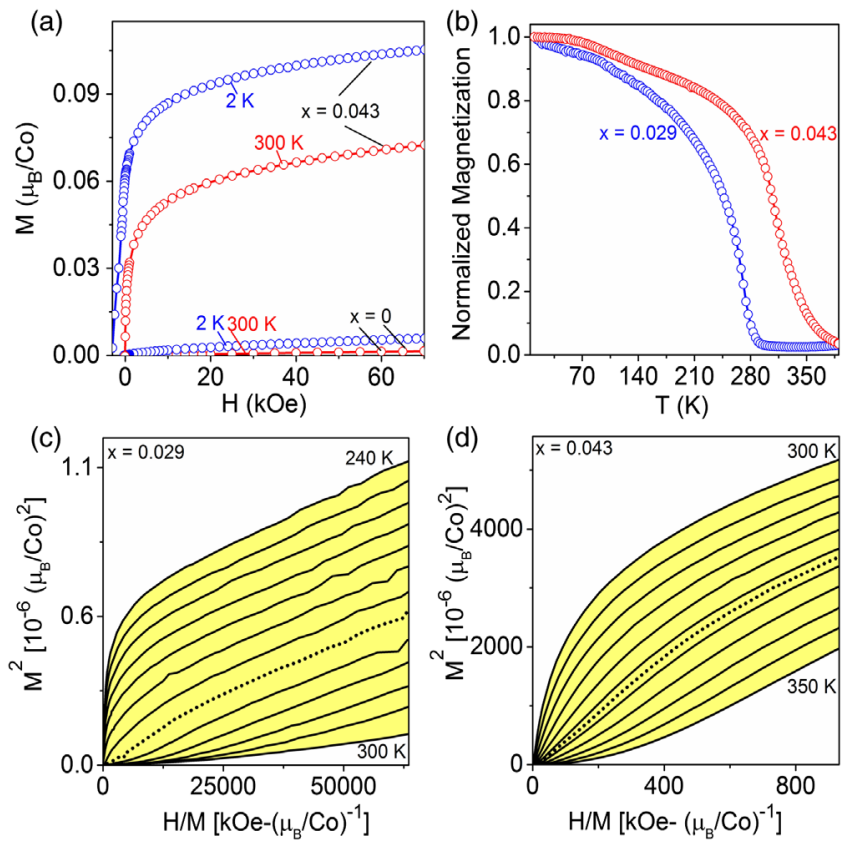

FIG. 3. Magnetic properties of $\mathrm{Co}_{1+x} \mathrm{Si}_{1-x}$ : (a) field dependence of the magnetization, (b) temperature dependence of the normalized magnetization in a field of $1 \mathrm{kOe}$, and (c),(d) Arrott plots. In the Arrott plots, the temperature interval between the solid lines is $5 \mathrm{~K}$. The dotted line in (d) corresponds to the measurement temperature $328 \mathrm{~K}$. 
ferromagnets [22-25] and low-temperature hysteresis are often observed as seen in $\mathrm{Co}_{1+x} \mathrm{Si}_{1-x}(x=0.029$ and 0.043 ) due to universal presence of some magnetocrystalline anisotropy [[24,25], Supplemental Material S0 and S3]. To obtain $T_{c}$, we have used mean-field Arrott plots, $M^{2}$ vs $H / M$ (Fig. 3), which yield $T_{c}=275 \mathrm{~K}$ for $x=$ 0.029 (c) and $328 \mathrm{~K}$ for $x=0.043$ (d).

To understand the onset of magnetic order in $\mathrm{Co}_{1+x} \mathrm{Si}_{1-x}$, we have carried out density-functional (DFT) calculations for several $x$ using supercells [see Supplemental Material S0]. Figure 4(a) shows a $3 \times 2 \times 1$ supercell with 48 atoms in which one $\mathrm{Si}$ atom was replaced by an excess-cobalt atom $\left(\mathrm{Co}^{\prime}\right)$ to yield $x=0.043$. Figure 4(b) schematically shows the average distance between the excess-Co atoms $\left(a_{\mathrm{eff}}\right)$ in $\mathrm{Co}_{1+x} \mathrm{Si}_{1-x}$. Figures 4(c)-4(f) show the density of states (DOS) for several Co concentrations. The identical spin-up ( $\uparrow)$ and spin-down $(\downarrow)$ bands for $x=0$ and 0.014 confirm the absence of spin polarization in these two alloys [Figs. 4(c)-4(d)]. There is, nevertheless, a qualitative difference between the two cases. CoSi is a semimetal close to the transition between diamagnetism and Pauli paramagnetism, with an overlap of valence and conduction bands by only about $30 \mathrm{meV}$ [26,27], a very small DOS and an intriguing new-fermion band structure near the Fermi level $[9-11,27] . \mathrm{Co}_{1.014} \mathrm{Si}_{0.986}$ is an ordinary metallic Pauli paramagnet, because the replacement of $\mathrm{Si}$ by $\mathrm{Co}$
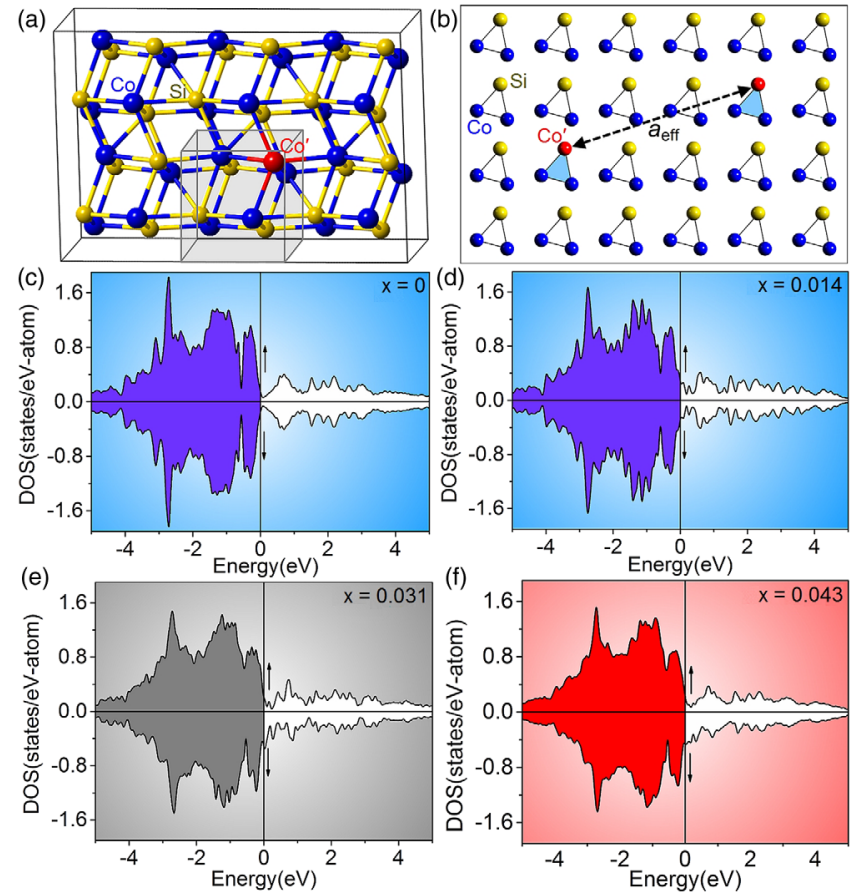

FIG. 4. Onset of magnetism in $\mathrm{Co}_{1+x} \mathrm{Si}_{1-x}$ : (a) $3 \times 2 \times 1$ supercell corresponding to $x=0.043$, where one $\mathrm{Si}$ atom is replaced with an excess-cobalt atom $\mathrm{Co}^{\prime}$ (red) and the gray region shows one B20 unit cell; (b) schematic location of the excess-Co atoms; (c)-(f) respective Co $3 d$ densities of states for the $x=0$, $0.014,0.031$, and 0.043 . reduces the number of valence electrons and shifts the Fermi level to the left.

Our calculations also show that the alloy having $x=$ 0.028 is nonmagnetic (not shown in Fig. 4). The $\uparrow$ and $\downarrow$ bands start splitting just below $x=0.031$ [Fig. 4(e)], where the moment is $0.13 \mu_{B}$ per Co atom (Fig. S4). The onset of the zero-temperature magnetism has the character of a magnetic quantum-phase transition (QPT) [28-32] above a critical excess-Co concentration $x_{c}=0.028$ (Fig. S4). For $x=0.043$ (red color scheme), the calculated moment is $0.18 \mu_{B}$ per $\mathrm{Co}$, in fair agreement with the experimental value of $0.11 \mu_{B} /$ Co. Note that our DFT calculations assume a ferromagnetic spin structure, ignoring noncollinear corrections. These corrections determine the periodicity $\lambda$ of spin spirals but have little effect on the onset of magnetic order, because $\lambda$ is normally much larger than the interatomic distance. Our DFT calculations show that, for $x=0.043$, the $\mathrm{Co}^{\prime}$ atom exhibits a moment of $1.71 \mu_{B}$ and somewhat spin-polarizes neighboring Co atoms, Fig. S5. The spin polarization decreases exponentially due to a consequence of the $k$ dependence of the susceptibility $[33,34]$, and this mechanism is discussed in Supplemental Material S5.

The chiral spin structure of B20 magnets is well known to support spin spirals (helices) [4] and skyrmions [1-3, 5,7]. Such noncollinear spin structures can be investigated by analyzing the field dependences of $M(H)$ and $\chi=$ $d M(H) / d H$, where they give rise to characteristic features or anomalies just below $T_{c}$ in single crystals, thin films and polycrystalline samples, corresponding to skyrmion spin structures $[3,35-45]$. The room-temperature field dependences of $M$ and $\chi$ for $x=0.043$ sample are shown in Fig. 5(a). $\chi(H)$ exhibits an anomaly between the fields $H_{A 1}=0.14$ and $H_{A 2}=0.32 \mathrm{kOe}$, similar to those observed for skyrmion spin structures in other systems [35-45]. Note that $M(H)$ data in Fig. 5(a) were taken at a close interval of 20 Oe on increasing $H$ for determining $\chi(H)$.

As observed in other systems [3,35-45], the skyrmion phase, green region in Fig. 5(b), is limited to a certain temperature range, between 285 and $305 \mathrm{~K}$ for $x=0.043$. Helimagnetism also involves two critical fields, $H_{c 1}$ and $H_{c 2}[8,43,46]$. The former refers to the orientation of the helix with respect to the crystal axes as indicated in Fig. 5(a). $H_{c 1}$ corresponds to the critical field at which $\chi$ increases rapidly in the low-field region $\left(H<H_{A 1}\right)$ and is determined from $d \chi / d H$ [42-44]. Note that $M(H)$ curves for helimagnetic materials measured upon increasing $H$ often show a kink corresponding to $H_{c 1}$ [45], which is also weakly seen in the case of the polycrystalline $\mathrm{Co}_{1.043} \mathrm{Si}_{0.957}$ as indicated by an arrow in Fig. 5(a). Below $H_{c 1}$, the spin structure is helical and above $H_{c 1}$, it is conical. Nevertheless, the skyrmion phase is often observed between helical and conical phases for a limited temperature range. Figure 5(c) shows the $H-T$ phase diagram, where red, green, and yellow regions correspond to helical, skyrmionic, and 

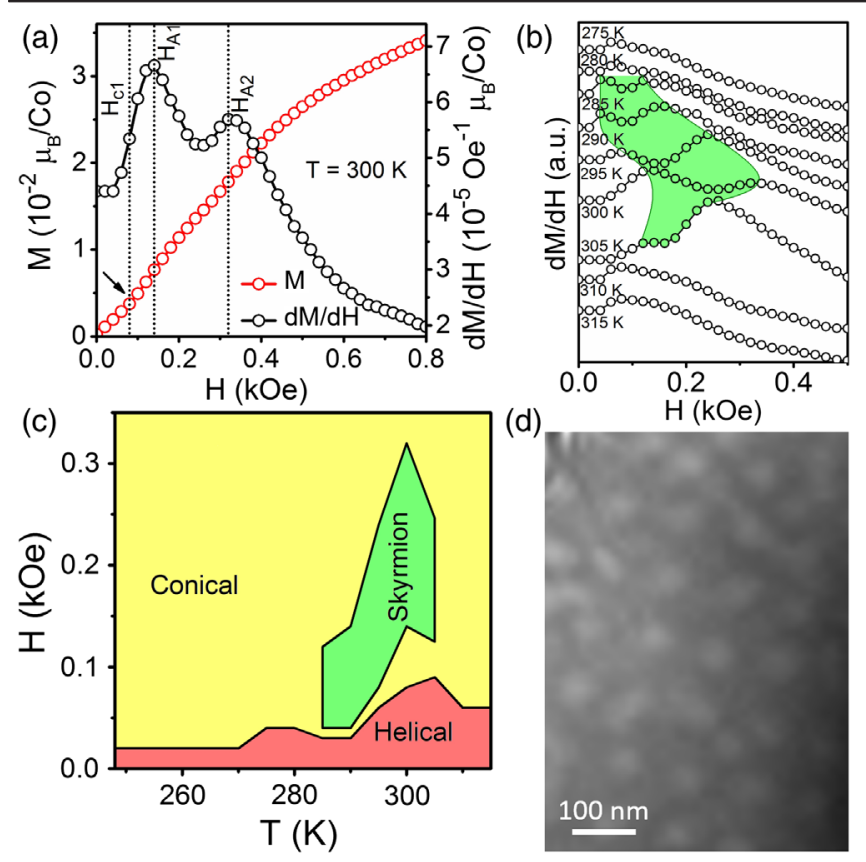

$$
A_{\mathrm{ex}}=\frac{3 k_{B} T_{c} S c^{\prime}}{2 a_{\mathrm{eff}}(S+1)},
$$

where $a_{\text {eff }}$ is the nearest-neighbor distance between the magnetic atoms [Fig. 4(b)], $c^{\prime}=1 / 6$ for CoSi and the spin quantum number $S$ is related to magnetization by $M_{s}=$ $g \mu_{B} S / V_{\text {eff }}[34,50] . a_{\text {eff }}$ depends on the excess-Co content and is about $8.07 \AA$ for $x=0.043$. Equation (2) gives $A_{\mathrm{ex}}=$ $0.07 \mu \mathrm{ergs} / \mathrm{cm}(0.7 \mathrm{pJ} / \mathrm{m})$ at $300 \mathrm{~K}$, which subsequently yields $\lambda=16 \mathrm{~nm}$. An alternative approach is to determine the exchange stiffness from Bloch's $T^{3 / 2}$ law [49], through $A_{\mathrm{ex}}=A_{\mathrm{SW}} M_{s} / 2 g \mu_{B}$, where $A_{S W}$ is the spin-wave stiffness. The Bloch law is limited to ferromagnets with a quadratic spin-wave dispersions $E \sim k^{2}$ and cannot be applied to the spin spirals, whose dispersion relation contains a term linear in $k$ [51]. However, close to saturation, the spins are nearly parallel and the negative curvature in the $M$ vs $T^{3 / 2}$ plot, caused by the linear term, is small (Fig. S7) and the Bloch law works quite well. For $x=0.043$ at room temperature, the Bloch analysis yields $A_{\mathrm{ex}}=$ $0.08 \mu \mathrm{ergs} / \mathrm{cm}(0.8 \mathrm{pJ} / \mathrm{m})$ and a helical periodicity of

FIG. 5. Micromagnetic analysis for $x=0.043$ : (a) roomtemperature field dependence of magnetization $M(H)$ and susceptibility $\chi=d M / d H$, (b) field dependence of $\chi$ at different temperatures, and (c) $H-T$ phase diagram for the bulk alloy. The anomaly between the fields $H_{A 1}$ and $H_{A 2}$ in (a) correspond to skyrmionic phase, whose temperature dependence is highlighted using a green-shaded region in (b). (d) The room-temperature Lorentz TEM image of a thin-film sample (see Supplemental Material S6 for additional details).

conical phases, respectively. We have obtained direct evidence for skyrmion structures through Lorentz TEM images, which show magnetic contrast corresponding to skyrmions around room temperature for a $\mathrm{Co}_{1.043} \mathrm{Si}_{0.957}$ thin film of about $50 \mathrm{~nm}$ thickness [Figs. 5(d) and S6].

$H_{c 2}$ describes the angular relationship of the spin inside the helix and corresponds to the field necessary to ensure parallel spin alignment $[8,43,46]$. The slow approach to saturation in the $M(H)$ curve of Fig. 3(a) provides information about the periodicity of the spin spiral. In the absence of DM interactions, the approach to saturation is a random-anisotropy effect [47], but the magnetocrystalline anisotropy is usually small in materials with cubic crystal structure (Supplemental Material S0) and cannot explain the incomplete saturation of the room-temperature $M(H)$ curve in the field range of 50-70 kOe in Fig. 3(a). Similarly, $H_{c 1}$ is small for CoSi as observed in other B20 magnets $[42,43]$ and therefore the approach to saturation is governed by $H_{c 2}$ through [48]

$$
H_{c 2}=\frac{2 A_{\mathrm{ex}} k^{2}}{\mu_{o} M_{s}} .
$$

Here, the helical wave vector $k=2 \pi / \lambda$ describes the periodicity $\lambda$ of the helix and $A_{\text {ex }}$ is the exchange stiffness. $A_{\text {ex }}$ can be estimated from $T_{c}$ through [49] $=17 \mathrm{~nm}$, which is smaller than the skyrmion size $(\sim 40 \mathrm{~nm})$ measured using Lorentz TEM. Room-temperature magnetocrystalline anisotropy is relatively low and can be safely excluded as a source of error in our estimate of $17 \mathrm{~nm}$, and the difference can be attributed to the difference in the dimensions of the samples used for Lorentz TEM and magnetometry measurements (Supplemental Material S8). Recently thin-film magnetostatic interactions have been shown to affect strongly the skyrmion size of B20 magnets [52]. The reason is that demagnetizing fields normally dominate the magnetocrystalline anisotropy of materials with cubic crystal structure [34] and become particularly important in thin films. Wang et al. address this point through $K=K_{u}-\mu_{o} M_{s}^{2} / 2$ [52]. Note that there is also a binary contrast between bulk B20 magnets (point group $T$ ) and multilayer systems (point groups $C_{n v}$ ), which is discussed in Supplemental Material S9.

The magnitude $\mathcal{D}$ of the DM interactions can be estimated by exploiting the well-known relation $\mathcal{D} \sim$ $k A_{S W}$ [43]. In more detail, adding the DM interaction for the point group $T$ of B20 structures to the interatomic exchange yields the energy density

$$
\eta=A_{\mathrm{ex}}(\nabla \boldsymbol{M})^{2}+\mathcal{D} \boldsymbol{M} \cdot(\nabla \times \boldsymbol{M}) .
$$

Here, $\mathcal{D}$ is the magnitude of diagonal magnetic gyration tensor, which is closely related but not equal to the magnitude of the atomic-scale DM vector (Supplemental Material S9). The Landau helices observed in B20 magnets are of the type

$$
\boldsymbol{M}=M_{s} \cos (k z) \boldsymbol{e}_{x}+M_{s} \sin (k z) \boldsymbol{e}_{y} .
$$

This expression transforms Eq. (3) into $\eta=k^{2} A_{\text {ex }}-\mathcal{D} k$. Minimization of $\eta$ with respect to $k, d \eta / d k=0$, yields 
$\mathcal{D}=2 A_{\mathrm{ex}} k$ and $\mathcal{D}=0.037 \mathrm{meV} / \AA^{2}$ for $x=0.043$. This value is small compared to most other B20 magnets, where $\mathcal{D}$ is larger than $1 \mathrm{meV} / \AA^{2}$ [43]. The smallness of $\mathcal{D}$ is a dilution effect. First, most of the Co atoms carry little or no magnetic moment and do not therefore contribute to $\mathcal{D}$. Second, on an atomic scale, the DM interactions involve products $\boldsymbol{S}_{C o^{\prime}} \times \boldsymbol{S}_{\mathrm{Co}}$, where $\boldsymbol{S}_{C o^{\prime}}$ is about $1.71 \mu_{B}$ but adjacent Co atoms have lower spins $S_{\mathrm{Co}}$ (about $0.4 \mu_{B}$, Fig. S5).

The estimated short periodicity $\lambda=17 \mathrm{~nm}$ of the spin spirals is striking. Since $\lambda$ is proportional to $A_{\mathrm{ex}} / \mathcal{D} \sim T_{c} / \mathcal{D}$, both the small $\mathcal{D}$ and the high $T_{c}$ lead to the expectation of long-wavelength spirals, which would be unfavorable from the viewpoint of nanoscale spin-electronics applications. In other words, the ordering temperature $T_{c}$ is proportional to the interatomic exchange $\mathcal{J}$, but so is $A_{\text {ex }}$, so that $T_{c}$ improvements are normally accompanied by unfavorable increase in skyrmion size. Our explanation involves the average distance $a_{\text {eff }}$ between neighboring spin-polarized excess-Co atoms, Fig. 4(b). In the $\mathrm{Co}_{1+x} \mathrm{Si}_{1-x}$ system, the $T_{c}$ value is relatively high $(>300 \mathrm{~K})$, while $\lambda$ scales as $\lambda \sim A_{\text {ex }} \sim \mathcal{J} / a_{\text {eff }}$, so that relatively large value of $a_{\text {eff }}$ in this dilute alloy near the QPT can lead to small values of $\lambda$.

In conclusion, we have fabricated B20-ordered $\mathrm{Co}_{1+x} \mathrm{Si}_{1-x}$ alloys that exhibit a quantum-phase transition from Pauli paramagnetism to a magnetically ordered state above $x_{c}=0.028$. For $x=0.043$, a magnetic-ordering temperature of $T_{c}=328 \mathrm{~K}$ has been achieved, which is the highest among known B20 magnets. The chirality of the crystals supports helical and skyrmionic spin structures near room temperature, and for $x=0.043$ a periodicity of about $17 \mathrm{~nm}$ and a Dzyaloshinski-Moriya interaction of $0.037 \mathrm{meV} / \AA^{2}$ have been theoretically estimated based on magnetization data. Lorentz TEM data indicates skyrmion radius of thin-film samples of about $20 \mathrm{~nm}$. The favorable combination of a high $T_{c}$ and a reasonably small $\lambda$ is a specific feature of the quantum-phase transition, which is realized through spin-polarized excess-Co atoms.

See Supplemental Material for additional details on methods, results, and discussion [18], which also includes Refs. [53-65].

This research is primarily supported by NSF-DMREF: SusChEM under Grant No. 1729288 (fabrication) and the U.S. Department of Energy under Award No. DE-FG0204ER46152 (characterization and theory). Research at Nebraska was performed in part in the Nebraska Nanoscale Facility: National Nanotechnology Coordinated Infrastructure and the Nebraska Center for Materials and Nanoscience, which are supported by the National Science Foundation under Grant No. NNCI1542182, and the Nebraska Research Initiative (NRI). Electron microscopy measurements at Cornell were supported by Platform for the Accelerated Realization, Analysis, and Discovery of Interface Materials
(PARADIM), an NSF MIP (DMR-1539918) and performed in electron microscope facilities, which are supported by CCMR, an NSF MRSEC (DMR-1719875). This work also used the Extreme Science and Engineering Discovery Environment (XSEDE) [allocation TG-PHY180014], which is supported by National Science Foundation Grant No. ACI-1548562. We thank Gregory David Fuchs and Emrah Turgut for helpful discussions.

*bbalasubramanian2@unl.edu

†dsellmyer@unl.edu

[1] N. Nagaosa and Y. Tokura, Nat. Nanotechnol. 8, 899 (2013).

[2] T. Nakajima, H. Oike, A. Kikkawa, E. P. Gilbert, N. Booth, K. Kakurai, Y. Taguchi, Y. Tokura, F. Kagawa, and T. Arima, T. Sci. Adv. 3, e1602562 (2017).

[3] A. S. Ahmed, J. Rowland, B. D. Esser, S. R. Dunsiger, D. W. McComb, M. Randeria, and R. K. Kawakami, Phys. Rev. Mater. 2, 041401(R) (2018).

[4] P. Bak and H. H. Jensen, J. Phys. C 13, L881 (1980).

[5] D. A. Gilbert, A. J. Grutter, P. M. Neves, G.-J. Shu, G. Zimanyi, B. B. Maranville, F.-C. Chou, K. Krycka, N. P. Butch, S. Huang, and J. A. Borchers, Phys. Rev. Mater. 3, 014408 (2019).

[6] H. D. Flack, Helv. Chim. Acta 86, 905 (2003).

[7] K. V. Shanavas and S. Satpathy, Phys. Rev. B 93, 195101 (2016).

[8] S. V. Grigoriev, A. S. Sukhanov, E. V. Altynbaev, S.-A. Siegfried, A. Heinemann, P. Kizhe, and S. V. Maleyev, Phys. Rev. B 92, 220415(R) (2015).

[9] Z. Rao, H. Li, T. Zhang, S. Tian, C. Li, B. Fu, C. Tang, L. Wang, Z. Li, W. Fan, J. Li, Y. Huang, Z. Liu, Y. Long, C. Fang, H. Weng, Y. Shi, H. Lei, Y. Sun, T. Qian, and H. Ding, Nature (London) 567, 496 (2019).

[10] D. S. Sanchez et al., Nature (London) 567, 500 (2019).

[11] D. Takane, Z. Wang, S. Souma, K. Nakayama, T. Nakamura, H. Oinuma, Y. Nakata, H. Iwasawa, C. Cacho, T. Kim, K. Horiba, H. Kumigashira, T. Takahashi, Y. Ando, and T. Sato, Phys. Rev. Lett. 122, 076402 (2019).

[12] S. M. Stishov, A. E. Petrova, V. A. Sidorov, and D. Menzel, Phys. Rev. B 86, 064433 (2012).

[13] N. E. Sluchanko, V. V. Glushkov, S. V. Demishev, A. A. Menovsky, L. Weckhuysen, and V. V. Moshchalkov, Phys. Rev. B 65, 064404 (2002).

[14] Y.-H. Liang, Sh.-Y. Yu, Ch.-L. Hsin, Ch.-W. Huang, and W.-W. Wu, J. Appl. Phys. 110, 074302 (2011).

[15] K. Seo, K. S. K. Varadwaj, P. Mohanty, S. Lee, Y. Jo, M.-H. Jung, J. Kim, and B. Kim, Nano Lett. 7, 1240 (2007).

[16] H. Okamoto, Phase Diagram of Binary Alloys (ASM, Materials Park, Ohio2000), p. 259.

[17] K. Ishida and T. Nishizawa, J. Phase Equilib. 12, 578 (1991).

[18] See Supplemental Material at http://link.aps.org/ supplemental/10.1103/PhysRevLett.124.057201 for additional details on methods, results, and discussion.

[19] S. V. Grigoriev, D. Chernyshov, V. A. Dyadkin, V. Dmitriev, E. V. Moskvin, D. Lamago, Th. Wolf, D. Menzel, J. Schoenes, S. V. Maleyev, and H. Eckerlebe, Phys. Rev. B 81, 012408 (2010). 
[20] A. Ullah, B. Balamurugan, W. Zhang, S. Valloppilly, X.-Z. Li, R. Pahari, L.-P. Yue, A. Sokolov, D. J. Sellmyer, and R. Skomski, IEEE Trans. Magn. 55, 7100305 (2019).

[21] S. V. Grigoriev, S.-A. Siegfried, E. V. Altynbayev, N. M. Potapova, V. Dyadkin, E. V. Moskvin, D. Menzel, A. Heinemann, S. N. Axenov, L. N. Fomicheva, and A. V. Tsvyashchenko, Phys. Rev. B 90, 174414 (2014).

[22] Y. Li, N. Kanazawa, X. Z. Yu, A. Tsukazaki, M. Kawasaki, M. Ichikawa, X. F. Jin, F. Kagawa, and Y. Tokura, Phys. Rev. Lett. 110, 117202 (2013).

[23] C. S. Spencer, J. Gayles, N. A. Porter, S. Sugimoto, Z. Aslam, C. J. Kinane, T. R. Charlton, F. Freimuth, S. Chadov, S. Langridge, J. Sinova, C. Felser, S. Blügel, Y. Mokrousov, and C. H. Marrows, Phys. Rev. B 97, 214406 (2018); A. Bauer, M. Garst, and C. Pfleiderer, Phys. Rev. B 93, 235144 (2016).

[24] S. X. Huang and C. L. Chein, Phys. Rev. Lett. 108, 267201 (2012); E. Karhu, S. Kahwaji, T. L. Monchesky, C. Parsons, M. D. Robertson, and C. Maunders, Phys. Rev. B 82, 184417 (2010).

[25] L. Lundgren, O. Beckman, V. Attia, S. P. Bhattacherjee, and M. Richardson, Phys. Scr. 1, 69 (1970).

[26] S. Asanabe, D. Shinoda, and Y. Sasaki, Phys. Rev. 134, A774 (1964).

[27] D. A. Pshenay-Severin, Y. V. Ivanov, A. A. Burkov, and A. T. Burkov, J. Phys. Condens. Matter 30, 135501 (2018).

[28] M. Nicklas, M. Brando, G. Knebel, F. Mayr, W. Trinkl, and A. Loydl, Phys. Rev. Lett. 82, 4268 (1999).

[29] R. Wang, A. Gebretsadik, S. Ubaid-Kassis, A. Schroeder, T. Vojta, P. J. Baker, F. L. Pratt, S. J. Blundell, T. Lancaster, I. Franke, J. S. Möller, and K. Page, Phys. Rev. Lett. 118, 267202 (2017).

[30] L. J. Bannenberg, F. Weber, A. J. E. Lefering, T. Wolf, and C. Pappas, Phys. Rev. B 98, 184430 (2018).

[31] A. Bauer, A. Neubauer, C. Franz, W. Münzer, M. Garst, and C. Pfleiderer, Phys. Rev. B 82, 064404 (2010).

[32] R. Pahari, B. Balasubramanian, R. Pathak, M.-C. Nguyen, S. R. Valloppilly, R. Skomski, A. Kashyap, C.-Z. Wang, K.-M. Ho, G. C. Hadjipanayis, and D. J. Sellmyer, Phys. Rev. B 99, 184438 (2019).

[33] W. Jones and N. H. March, Theoretical Solid State Physics I (Wiley \& Sons, London, 1973).

[34] R. Skomski, Simple Models of Magnetism (University Press, Oxford 2008).

[35] A. Bauer and C. Pfleiderer, Phys. Rev. B 85, 214418 (2012).

[36] M. N. Wilson, E. A. Karhu, A. S. Quigley, U. K. Rößler, A. B. Butenko, A. N. Bogdanov, M. D. Robertson, and K. L. Monchesky, Phys. Rev. B 86, 144420 (2012).

[37] C. Dhital, L. DeBeer-Schmitt, D. P. Young, and J. F. DiTusa, Phys. Rev. B 99, 024428 (2019).

[38] E. Ruff, P. Lunkenheimer, A. Loidi, H. Berger, and S. Krohns, Sci. Rep. 5, 15025 (2015).

[39] Y. Tokunaga, X. Z. Yu, J. S. White, H. M. Rønnow, D. Morikawa, Y. Taguchi, and Y. Tokura, Nat. Commun. 6, 7638 (2015).

[40] S. S. Samatham and V. Ganesan, Phys. Status Solidi B 252, 1810 (2015).
[41] Y. Nii, T. Nakajima, A. Kikkawa, Y. Yamasaki, K. Ohishi, J. Suzuki, Y. Taguchi, T. Arima, Y. Tokura, and Y. Iwasa, Nat. Commun. 6, 8539 (2015).

[42] A. Bauer and C. Pfleiderer, Generic Aspects of Skyrmion Lattices in Chiral magnets in Topological Structures in Ferroic Materials: Domain Walls, Vortices and Skyrmions, edited by J. Saidel, Springer Series in Material Science Vol. 228 (Springer International Publishing, Switzerland, 2016), pp. 1-28.

[43] C. Dhital, L. DeBeer-Schmitt, Q. Zhang, W. Xie, D. P. Young, and J. F. DiTusa, Phys. Rev. B 96, 214425 (2017).

[44] H. C. Wu, T. Y. Wei, K. D. Chandrasekhar, T. Y. Chen, H. Berger, and H. D. Yang, Sci. Rep. 5, 13579 (2015); M. Garst, J. Waizner, and D. Grunder, J. Phys. D 50, 293002 (2017).

[45] S. V. Grigoriev, V. A. Dyadkin, D. Menzel, J. Schoenes, Yu. O. Chetverikov, A. I. Okorokov, H. Eckerlebe, and S. V. Maleyev, Phys. Rev. B 76, 224424 (2007).

[46] S.-A. Siegfried, A. S. Sukhanov, E. V. Altynbaev, D. Honecker, A. Heinemann, A. V. Tsvyashchenko, and S. V. Grigoriev, Phys. Rev. B 95, 134415 (2017).

[47] G. Hadjipanayis, D. J. Sellmyer, and B. Brandt, Phys. Rev. B 23, 3349 (1981).

[48] E. Turgut, A. Park, K. Nguyen, A. Moehle, D. A. Muller, and G. D. Fuchs, Phys. Rev. B 95, 134416 (2017).

[49] H. Kronmüller and M. Fähnle, Micromagnetism and the Microstructure of Ferromagnetic Solids (University Press, Cambridge 2003).

[50] R. Skomski and J. M. D. Coey, Permanent Magnetism (Institute of Physics, Bristol 1999).

[51] R. L. Melcher, Phys. Rev. Lett. 30, 125 (1973).

[52] X. S. Wang, H. Y. Yuan, and X. R. Wang, Commun. Phys. 1, 31 (2018).

[53] B. Balamurugan, B. Das, W. Y. Zhang, R. Skomski, and D. J. Sellmyer, J. Phys. Condens. Mater. 26, 064204 (2014).

[54] G. Kresse and J. Furthmüller, Phys. Rev. B 54, 11169 (1996).

[55] J. P. Perdew, K. Burke, and M. Ernzerhof, Phys. Rev. Lett. 77, 3865 (1996).

[56] G. Kresse and D. Joubert, Phys. Rev. B 59, 1758 (1999).

[57] H. J. Monkhorst and J. D. Pack, Phys. Rev. B 13, 5188 (1976).

[58] D. Hobbs, G. Kresse, and J. Hafner, Phys. Rev. B 62, 11556 (2000).

[59] E. R. Callen and H. B. Callen, Phys. Rev. 129, 578 (1963).

[60] https://www.unl.edu/ncmn-enif/xzli/computer-programs.

[61] D. S. Fisher, Phys. Rev. Lett. 69, 534 (1992).

[62] X. Z. Yu, Y. Onose, N. Kanazawa, J. H. Park, J. H. Han, Y. Matsui, N. Nagaosa, and Y. Tokura, Nature 465, 901 (2010).

[63] E. A. Karhu, S. Kahwaji, M. D. Robertson, H. Fritzsche, B. J. Kirby, C. F. Majkrzak, and T. L. Monchesky, Phys. Rev. B 84, 060404(R) (2011).

[64] S. Mühlbauer, B. Binz, F. Jonietz, C. Pfleiderer, A. Rosch, A. Neubauer, R. Georgii, and P. Böni, Science 323, 915 (2009).

[65] X. Z. Yu, Y. Onose, N. Kanazawa, J. H. Park, J. H. Han, Y. Matsui, N. Nagaosa, and Y. Tokura, Nature (London) 465, 901 (2010). 